\title{
Asymptotic Behavior of the Solution of the Distribution Diffusion Equation for FENE Dumbbell Polymer Model
}

\author{
I. S. Ciuperca ${ }^{1}$ and L. I. Palade ${ }^{2 *}$ \\ ${ }^{1}$ Université de Lyon, Université Lyon 1, Institut Camille Jordan, UMR 5208 CNRS \\ 69622 Villeurbanne, France \\ ${ }^{2}$ Université de Lyon, INSA de Lyon, Institut Camille Jordan \\ UMR 5208 CNRS \& Pôle de Mathématiques, 69621 Villeurbanne, France
}

\begin{abstract}
This paper deals with the evolution Fokker-Planck-Smoluchowski configurational probability diffusion equation for the FENE dumbbell model in dilute polymer solutions. We prove the exponential convergence in time of the solution of this equation to a corresponding steady-state solution, for arbitrary velocity gradients.
\end{abstract}

Key words: FENE dumbbell chains, Fokker-Planck-Smoluchowski equation, entropy function, sectorial operators

AMS subject classification: 35K10, 35K15, 35B35

\section{Introduction}

Molecular interactions govern the rheological behavior of non-Newtonian and viscoelastic fluids. One way to account for is offered by the kinetical theory initiated by Kirkwood [13] and significantly enriched by Bird, Curtiss, Armstrong and Hassager (see their cornerstone work [3]). Within the aforementioned conceptual framework, polymer chains are modeled as a necklace made up of freely jointed beads and springs. Among the simplest models is the elastic dumbbell aka FENE chain (basically two beads connected by a Finitely Extensible Nonlinear Elastic spring - hence the acronym) discovered by Warner and Bird during late sixties (see page 76 and subsequent in [3]).

${ }^{*}$ Corresponding author. E-mail: liviu-iulian.palade@insa-lyon.fr 
It has been found very succesful in modeling certain shear/extensional flows of dilute polymer solutions.

At the hardcore of any kinetical model of viscoelasticity there is a configurational probability diffusion equation of Fokker-Planck-Smoluchowski type. Solving it (at least in principle) allows for calculating the stress tensor that is necessary for the study of momentum balance equations.

The problem under consideration is the following (the notations are those of [7]):

Find $\tilde{\psi}(\tilde{q}, \tilde{t}) \geq 0$, with $\tilde{q} \in B(0, \tilde{\delta})$ and $\tilde{t} \geq 0$, solution to the FENE configurational probability diffusion equation, which in non-dimensional form is given by:

$$
\frac{\partial \tilde{\psi}}{\partial \tilde{t}}-\nabla_{\tilde{q}} \cdot\left(-\tilde{\theta} \tilde{q} \tilde{\psi}+\frac{1}{2 \mathrm{De}} \nabla_{\tilde{q}} \tilde{\psi}+\frac{1}{2 \mathrm{De}} \tilde{F} \tilde{\psi}\right)=0
$$

compatible with boundary and initial conditions

$$
\left.\left(-\tilde{\theta} \tilde{q} \tilde{\psi}+\frac{1}{2 \mathrm{De}} \nabla_{\tilde{q}} \tilde{\psi}+\frac{1}{2 \mathrm{De}} \tilde{F} \tilde{\psi}\right) \cdot \tilde{q}\right|_{\partial B(0, \tilde{\delta})}=0
$$

and

$$
\tilde{\psi}(\tilde{q}, \tilde{t}=0)=\tilde{\psi}_{0}(\tilde{q})
$$

In the above $\tilde{q}$ is the dumbell end-to-end vector, $\tilde{\delta}>0$ is a fluid depending parameter, $B(0, \tilde{\delta}) \subset$ $\mathbb{R}^{n}$ is an open ball centered at 0 of radius $\tilde{\delta}$, De is the Deborah number and $\tilde{\theta}$ denotes the macroscopic velocity gradient (which is traceless, $\operatorname{tr}(\tilde{\theta})=0$, due to incompressibility (see [2], [11], [14], [19]). $\tilde{F}=\frac{\tilde{q}}{1-(\|\tilde{q}\| / \tilde{\delta})^{2}}$ is the Warner-Bird's (molecular connector) elastic force (see [3] as well as [14] and [17]).

Further writing simplification is achieved upon carrying out the variable changes $q=\tilde{q} / \tilde{\delta}$, $t=\tilde{t} /\left(2 \operatorname{De} \tilde{\delta}^{2}\right)$. With the following notations: $D=B(0,1), \psi(t, q)=\tilde{\psi}(\tilde{t}, \tilde{q}), \theta=2 \operatorname{De} \tilde{\delta}^{2} \tilde{\theta}$, $\delta=\tilde{\delta}^{2} / 2, \Pi(q)=-\log \left(1-\|\tilde{q}\|^{2}\right), \psi_{0}(q)=\tilde{\psi}_{0}(\tilde{q})$, the initial boundary value problem (IBVP) now looks:

$$
\begin{gathered}
\frac{\partial \psi}{\partial t}-\nabla_{q} \cdot\left[-\theta q \psi+\nabla_{q} \psi+\delta\left(\nabla_{q} \Pi\right) \psi\right]=0 \\
{\left.\left[-\theta q \psi+\nabla_{q} \psi+\delta\left(\nabla_{q} \Pi\right) \psi\right] \cdot q\right|_{q \in \partial D}=0} \\
\left.\psi(q, t=0)\right|_{q \in D}=\psi_{0}(q) .
\end{gathered}
$$

We now have that

$$
\nabla_{q} \psi+\delta \nabla_{q}(\Pi) \psi=e^{-\delta \Pi} \nabla_{q}\left(e^{\delta \Pi} \psi\right)=M \nabla_{q}\left(\frac{\psi}{M}\right)
$$


where $M(q)=\left(1-\|q\|^{2}\right)^{\delta}$. Therefore, equations (1.4)-(1.5) can be re-written as:

$$
\begin{gathered}
\frac{\partial \psi}{\partial t}-\nabla_{q} \cdot\left[M \nabla_{q}\left(\frac{\psi}{M}\right)\right]+\nabla_{q} \cdot(\theta q \psi)=0 \\
\left.\left\{-M \nabla_{q}\left(\frac{\psi}{M}\right)+\theta \psi\right\}\right|_{\partial D}=0 .
\end{gathered}
$$

We will assume throughout this work that $\delta>2$, which is physically realistic.

Let $L_{M}^{2}$ and $H_{M}^{1}$ denote the spaces

$$
\begin{gathered}
L_{M}^{2}:=\left\{u \in L_{\mathrm{loc}}^{1}(D) \text { s.t. } \int_{D} \frac{u^{2}}{M} \mathrm{~d} q<\infty\right\} \\
H_{M}^{1}:=\left\{u \in L_{M}^{2} \text { s.t. } \int_{D} \frac{u^{2}}{M}+M\left[\nabla_{q}\left(\frac{u}{M}\right)\right]^{2} \mathrm{~d} q<\infty\right\} .
\end{gathered}
$$

As $\tilde{\psi}_{0}, \tilde{\psi}(\cdot, t)$ are probability densities, it is compulsory that

$$
\begin{gathered}
\psi_{0} \geq 0, \int_{D} \psi_{0} \mathrm{~d} q=a=\frac{1}{\tilde{\delta}^{n}} \\
\psi(\cdot, t) \geq 0, \int_{D} \psi(q, t) \mathrm{d} q=a .
\end{gathered}
$$

By integrating (1.7) and using (1.8) one gets $\frac{d}{d t} \int_{D} \psi \mathrm{d} q=0$. Therefore the condition (1.12) is satisfied provided the initial data satisfy (1.11). Moreover, invoking the maximum principle one obtains $\psi(\cdot, t) \geq 0$ provided that $\psi_{0} \geq 0$.

Let us now focus on a time independent (steady-state) solution $\psi_{\infty}$ to the below given problem:

$$
\begin{gathered}
-\nabla_{q} \cdot\left[M \nabla_{q}\left(\frac{\psi_{\infty}}{M}\right)\right]+\nabla_{q} \cdot\left(\theta q \psi_{\infty}\right)=0, \text { on } D \\
\left.\left\{-M \nabla_{q}\left(\frac{\psi_{\infty}}{M}\right)+\theta \psi_{\infty}\right\}\right|_{\partial D}=0 \\
\psi_{\infty} \geq 0 \\
\int_{D} \psi_{\infty} \mathrm{d} q=1
\end{gathered}
$$

We now state the variational formulation of the problem (1.13)-(1.16): find $\psi_{\infty} \in H_{M}^{1}$, satisfying (1.15)-(1.16) and such that

$$
\int_{D}\left[M \nabla_{q}\left(\frac{\psi_{\infty}}{M}\right) \cdot \nabla_{q}\left(\frac{\varphi}{M}\right)-\theta q \cdot \nabla_{q}\left(\frac{\varphi}{M}\right)\right] \mathrm{d} q=0, \quad \forall \varphi \in H_{M}^{1}
$$


We proved in [7] the existence and uniqueness of a solution to (1.17), $\psi_{\infty} \in \mathscr{C}^{0}(\bar{D})$, and that there exists $0<\alpha_{0} \leq \alpha_{1}$ such that $\alpha_{0} M \leq \psi_{\infty}(q) \leq \alpha_{1} M, \forall q \in D$.

Let the following Banach space be given by (see [7]):

$$
X_{\beta}:=\left\{\varphi \in \mathscr{C}^{0}(\bar{D}), \text { s.t. } \sup _{q \in D} \frac{|\varphi(q)|}{(M(q))^{\beta}}<\infty\right\}, \beta \geq 0
$$

endowed with the norm

$$
\|\varphi\|_{X_{\beta}}=\sup _{q \in D} \frac{|\varphi(q)|}{(M(q))^{\beta}}
$$

and let the cone of non-negative functions in $X_{\beta}$ be given by $P_{\beta}=\left\{\varphi \in X_{\beta}\right.$ s.t. $\left.\varphi \geq 0\right\}$. Clearly the interior $\stackrel{\circ}{P}_{\beta}$ of $P_{\beta}$, in $X_{\beta}$, is given by

$$
\begin{aligned}
\stackrel{\circ}{P}_{\beta}= & \left\{\varphi \in X_{\beta} \text { s.t. } \exists \alpha>0, \varphi(q) \geq \alpha[M(q)]^{\beta}, \quad \forall q \in D\right\} \\
= & \left\{\varphi \in \mathscr{C}^{0}(\bar{D}), \exists \alpha_{1}, \alpha_{2}, 0<\alpha_{1} \leq \alpha_{2} \text { s.t. } \alpha_{1}[M(q)]^{\beta} \leq \varphi(q) \leq \alpha_{2}[M(q)]^{\beta},\right. \\
& \forall q \in D\} .
\end{aligned}
$$

It is now manifest that $\psi_{\infty} \in \stackrel{\circ}{P}_{1}$. Actually the solution $\psi_{\infty}$ is an eigenvector corresponding to eigenvalue 0 of the operator $L: H_{M}^{1} \rightarrow\left(H_{M}^{1}\right)^{\prime}$ defined, for any $u, \varphi \in H_{M}^{1}$, as:

$$
\langle L(u), \varphi\rangle=\int_{D}\left[M \nabla_{q}\left(\frac{u}{M}\right) \cdot \nabla_{q}\left(\frac{\varphi}{M}\right)-\theta q u \cdot \nabla_{q}\left(\frac{\varphi}{M}\right)\right] \mathrm{d} q .
$$

The proof of the existence and uniqueness of the solution $\psi_{\infty}$ given in [7] is based on the use of the Krein-Rutman's theorem when working on the spaces $L_{M}^{2}$ and $X_{1}$, respectively.

In this paper we prove that the solution $\psi$ to the IBVP (1.7), (1.8), and (1.6) is given as the semigroup associated to the operator $L$ which acts on some apropiate spaces (to be defined later on). We also prove the exponential convergence in time of $\psi$ to the steady state solution $a \psi_{\infty}$, a fact physically sound but for which no full proof has been offered until now.

The issue of existence/uniqueness of solutions $\psi(t, q)$ to the diffusion equation of the FENE model for general flows of practical interest has been addressed in Du, Liu, Yu [9], Jourdain, LeBris, LeLièvre, Otto [12], Masmoudi [16], Chupin [6] and Ciuperca and Palade [7]. For some recent results on a related probability diffusion equation for rigid dumbbell polymers see Ciupercă and Palade [8] and on a Fokker-Planck model in computational neuroscience see Carillo, Cordier, Mancini [5].

Moreover, a lot of attention has been devoted to the Fokker-Planck-Smoluchowski equation coupled with the momentum balance equation, system solved by the velocity and the pressure of 
the fluid: see for example the work by Zhang, Zhang [21], Lin, Zhang, Zhang [15], Barret, Schwab, Sülli [1], and Busuioc, Ciuperca, Iftimie and Palade [4] (and references cited therein).

In [12] the authors have shown that $\psi$ tends to $a \psi_{\infty}$ for $t \rightarrow+\infty$, for some particular flows, i.e. for symmetric velocity gradients $\theta=\theta^{T}$, antisymmetric $-\theta=\theta^{T}$, and for flows for which the symmetric part of $\theta$ is small enough. Here, we extend the results of Jourdain et al. and offer a proof of the convergence for arbitrary velocity gradients. Moreover, we prove that $\psi$ converges exponentially to $a \psi_{\infty}$ not only w.r.t. the $L^{1}$ norm (as was shown in [12]) but also w.r.t. stronger norms.

In Section 2. we give several important properties of the operator $L$ and in Section 3. the result of convergence of the solution $\psi$.

\section{Properties of operator $L$}

Let us now consider the operator $L_{0}(\varphi)=-\nabla \cdot\left[M \nabla\left(\frac{\varphi}{M}\right)\right]$ on the space $L_{M}^{2}$, with domain

$$
D\left(L_{0}\right)=\left\{\varphi \in H_{M}^{1} \text { s.t. } \nabla \cdot\left[M \nabla\left(\frac{\varphi}{M}\right)\right] \in L_{M}^{2},\left.M \nabla\left(\frac{\varphi}{M}\right) \cdot q\right|_{\partial D}=0\right\} .
$$

In the above, the condition $\left.M \nabla\left(\frac{\varphi}{M}\right) \cdot q\right|_{\partial D}=0$ is to be understood in the following weak sense: $\int_{D} \frac{\phi}{M} L_{0}(\varphi) \mathrm{d} q=\int_{D} M \nabla\left(\frac{\varphi}{M}\right) \cdot \nabla\left(\frac{\phi}{M}\right) \mathrm{d} q, \quad \forall \phi \in H_{M}^{1}$. Since $\mathcal{D}(D)$ is dense in $L_{M}^{2}$, we have that $D\left(L_{0}\right)$ is dense in $L_{M}^{2}$ (see [16]). Clearly $L_{0}$ is symmetric and non-negative operator. We deduce (see [10]) that $L_{0}$ is a sectorial operator.

The operator $L$ defined in (1.20) can be considered as an operator defined on $L_{M}^{2}$ with domain $D(L)=D\left(L_{0}\right)$ and given by $L \varphi=-\nabla \cdot\left[M \nabla\left(\frac{\varphi}{M}\right)\right]+\nabla \cdot(\theta q \varphi)$. We have the following result:

Lemma 2..1. The operator $L$ is sectorial.

Proof. Write $L$ as $L=L_{0}+\mathrm{Id}+B$, with Id standing for the identity operator and $B(\varphi)=$ $-\varphi+\nabla \cdot(\theta q \varphi)$. First observe that $L=L_{0}+$ Id is sectorial. Next, we show that

$$
B \in \mathscr{L}\left(H_{M}^{1}, L_{M}^{2}\right)
$$

Proving the above statement simmers down to showing that, for any $\varphi \in H_{M}^{1}$, $\|\nabla \cdot(\theta q \varphi)\|_{L_{M}^{2}} \leq$ $c_{1}\|\varphi\|_{H_{M}^{1}}$, with $c_{1} \geq 0$ a constant. Now, since $\nabla \cdot(\theta q)=0$, one has

$$
\nabla \cdot(\theta q \varphi)=\nabla \cdot\left(M \theta q \frac{\varphi}{M}\right)=M \theta q \cdot \nabla\left(\frac{\varphi}{M}\right)+\nabla M \cdot \theta q \frac{\varphi}{M}
$$

However,

$$
\left\|M \theta q \cdot \nabla\left(\frac{\varphi}{M}\right)\right\|_{L_{M}^{2}}=\left\|\theta q \sqrt{M} \cdot \nabla\left(\frac{\varphi}{M}\right)\right\|_{L^{2}(D)} \leq\|\theta\|\|\varphi\|_{H_{M}^{1}}
$$


and

$$
\left\|\nabla M \cdot \theta q \frac{\varphi}{M}\right\|_{L_{M}^{2}}=\left\|2 \delta \theta q \cdot q \frac{\varphi}{M^{1 / 2+1 / \delta}}\right\|_{L^{2}(D)} \leq 2 \delta\|\theta\|\left\|\frac{\varphi}{M^{1 / 2+1 / \delta}}\right\|_{L^{2}(D)}
$$

Now, as a consequence of Theorem 6.2.5 in [18] (see also the inclusion 3.10 in [7]), the following inequality holds true:

$$
\left\|\frac{\varphi}{M^{1 / 2+1 / \delta}}\right\|_{L^{2}(D)} \leq c_{2}\|\varphi\|_{H_{M}^{1}}, \forall \varphi \in H_{M}^{1}
$$

Then (2.2)-(2.4) lead to (2.1). We deduce from (2.1) that

$$
D(B) \supset D\left(L_{0}+\mathrm{Id}\right)=D\left(L_{0}\right)
$$

Let $\varphi \in D\left(L_{0}\right)$ and $f=\left(L_{0}+\mathrm{Id}\right)(\varphi) \in L_{M}^{2}$. Consider now the variational formulation of the latest equality; it looks:

$$
\int_{D}\left[M \nabla\left(\frac{\varphi}{M}\right) \cdot \nabla\left(\frac{\xi}{M}\right)+\frac{\varphi \xi}{M}\right] \mathrm{d} q=\int_{D} \frac{f \xi}{M} \mathrm{~d} q, \quad \forall \xi \in H_{M}^{1}
$$

Taking $\xi=\varphi$ leads to

$$
\|\varphi\|_{H_{M}^{1}}^{2}=\left\langle\left(L_{0}+\mathrm{Id}\right) \varphi, \varphi\right\rangle_{L_{M}^{2}} \leq\left\|\left(L_{0}+\mathrm{Id}\right) \varphi\right\|_{L_{M}^{2}}\|\varphi\|_{L_{M}^{2}}
$$

We deduce that, for any $\epsilon>0$,

$$
\|\varphi\|_{H_{M}^{1}} \leq \sqrt{\left\|\left(L_{0}+\mathrm{Id}\right) \varphi\right\|_{L_{M}^{2}}} \sqrt{\|\varphi\|_{L_{M}^{2}}} \leq \epsilon\left\|\left(L_{0}+\mathrm{Id}\right) \varphi\right\|_{L_{M}^{2}}+\frac{1}{4 \epsilon}\|\varphi\|_{L_{M}^{2}}
$$

The result stated in Lemma $2 . .1$ is now a consequence of the above inequality and of Theorem 1.3.2 in [10].

Back now to the evolution problem as given in equations (1.7), (1.8), (1.6). The boundary condition (1.8) is to be understood in the following weak sense: for $\psi \in D\left(L_{0}\right)$ and for any $\xi \in H_{M}^{1}$,

$$
\begin{aligned}
& \int_{D}\left\{-\nabla_{q} \cdot\left[M \nabla_{q}\left(\frac{\psi}{M}\right)\right]+\nabla_{q}(\theta q \psi)\right\} \frac{\xi}{M} \mathrm{~d} q= \\
& \int_{D}\left[M \nabla_{q}\left(\frac{\psi}{M}\right) \cdot \nabla_{q}\left(\frac{\xi}{M}\right)-\theta q \psi \cdot \nabla_{q}\left(\frac{\xi}{M}\right)\right] \mathrm{d} q
\end{aligned}
$$

In fact the above equality is satisfied for any $\psi \in D\left(L_{0}\right)$. To get a grasp of this, observe first that (2.6) holds true for any $\xi \in \mathscr{D}(D)$. As $\mathscr{D}(D)$ is dense in $H_{M}^{1}$ (see e.g. Remark 37 in [16]) one sees (2.6) is solved for any $\psi \in D\left(L_{0}\right)$.

Then the evolution problem (1.7), (1.8), (1.6) can be re-stated as: find $\psi:\left[0,+\infty\left[\rightarrow L_{M}^{2}\right.\right.$, $\psi(t) \in D(L) \forall t>0$, such that: 


$$
\begin{aligned}
& \frac{\partial \psi}{\partial t}+L \psi=0, \quad t>0 \\
& \psi(t=0)=\psi_{0}, \quad \psi_{0} \in L_{M}^{2}, \text { given. }
\end{aligned}
$$

From Lemma (2..1) we gather that there exists an analytical semigroup $e^{-t L}$ spanned by the operator $-L$; then $\psi(t)=e^{-t L} \psi_{0}$ is no other than the solution to (2.7) (see [10]).

Another important property of $L$ (the proof of which is presented in [7]) is:

Lemma 2..2. There exists $\alpha>0$ s.t. $L+\alpha$ Id is invertible from $H_{M}^{1}$ to $\left(H_{M}^{1}\right)^{\prime}$.

Let us now prove the following:

Lemma 2..3. $L$ is a closed operator from $D(L)$ to $L_{M}^{2}$.

Proof. Let $\varphi_{n} \in D(L)$ and $\varphi \in L_{M}^{2}$, with $\varphi_{n} \underset{n \rightarrow+\infty}{\stackrel{L_{M}^{2}}{\longrightarrow}} \varphi$. Let $f_{n}=L\left(\varphi_{n}\right), f \in L_{M}^{2}$, such that $f_{n} \underset{n \rightarrow+\infty}{\stackrel{L_{M}^{2}}{\longrightarrow}} f$. Then $L\left(\varphi_{n}\right)+\alpha \varphi_{n}=f_{n}+\alpha \varphi_{n}$. From Lemma (2..2) one deduces $\varphi_{n}$ is a Cauchy sequence in $H_{M}^{1}$. This implies $f_{n} \underset{n \rightarrow+\infty}{\stackrel{H_{M}^{1}}{\longrightarrow}} \varphi$, hence $\varphi \in H_{M}^{1}$. Let now $n \rightarrow+\infty$ in the below equality:

$$
\int_{D}\left[M \nabla\left(\frac{\varphi_{n}}{M}\right) \cdot \nabla\left(\frac{\xi}{M}\right)-\theta q \varphi_{n} \cdot \nabla\left(\frac{\xi}{M}\right)\right] \mathrm{d} q=\int_{D} \frac{f_{n} \xi}{M} \mathrm{~d} q, \forall \xi \in H_{M}^{1}
$$

Carrying out the limit calculations leads to:

$$
\int_{D}\left[M \nabla\left(\frac{\varphi}{M}\right) \cdot \nabla\left(\frac{\xi}{M}\right)-\theta q \varphi \cdot \nabla\left(\frac{\xi}{M}\right)\right] \mathrm{d} q=\int_{D} \frac{f \xi}{M} \mathrm{~d} q, \forall \xi \in H_{M}^{1} .
$$

This implies $\varphi \in D(L)$ and $L(\varphi)=f$, which ends the proof.

It is clear from Lemma (2..2) that $-\alpha$ is an element of the resolvent set of $L$ (with $L$ being seen from $D(L)$ onto $L_{M}^{2}$ ). From the compact embedding of $H_{M}^{1}$ into $L_{M}^{2}$ (see [18]) we deduce that $L$ has a compact resolvent in $L_{M}^{2}$. From Lemma (2..3) one concludes the spectrum $\sigma(L)$ of $L$ consists only of isolated eigenvalues with finite multiplicity.

\section{Convergence properties of the solution $\psi$}

Recall $\psi_{\infty}$ is a solution to the steady-state problem (1.13)-(1.16); $\psi_{\infty}$ it is also an eigenvector of $L$ corresponding to the simple eigenvalue 0 of $L$.

Let us now introduce the function $A$ given by

$$
A(q)=\frac{\psi_{\infty}(q)}{M(q)}
$$


From the fact that $\psi_{\infty} \in \stackrel{\circ}{P}_{1}$ we deduce that we can introduce the positive real numbers

$$
A^{*} \equiv \sup _{q \in D} A(q)>0 \quad \text { and } \quad A_{*} \equiv \inf _{q \in D} A(q)>0 .
$$

Remark also that $\log (A)$ is a bounded function.

We now give the following Sobolev logarithmic inequality:

\section{Lemma 3..1.}

$$
\int_{D} \Phi \log \left(\frac{\Phi}{\psi_{\infty}}\right) d q \leq s \int_{D}\left|\nabla\left(\frac{\Phi}{\psi_{\infty}}\right)\right|^{2} \frac{\psi_{\infty}^{2}}{\Phi} d q, \quad \forall \Phi \in H_{M}^{1} \cap \stackrel{\circ}{P}_{1}
$$

with $s=\frac{1}{4 \delta}\left(\frac{A^{*}}{A_{*}}\right)^{2}$.

Proof. Remark first that for $\theta=0$ the stationary solution $\psi_{\infty}$ is given by

$$
\psi_{\infty}=\frac{M}{\int_{D} M(q) d q} .
$$

Let us introduce the real function defined on $]-1,1\left[\right.$ by $g(r)=-\delta \log \left(1-r^{2}\right)$. We easily verify that $g^{\prime \prime} \geq 2 \delta$ which proves that $-\log \psi_{\infty}$ is a $2 \delta$ - convex function in the case $\theta=0$. We deduce from equation (43) in [12] that (3.2) is verified with $s=\frac{1}{4 \delta}$ in the case $\theta=0$.

For a general velocity gradient $\theta$ we shall use the result of Lemma 1 in [12]. We have from (3.1)

$$
\psi_{\infty}=\frac{M}{\int_{D} M(q) d q} \exp (\hat{\Pi}(q))
$$

with

$$
\hat{\Pi}=\log \left(\int_{D} M\right)+\log (A) .
$$

Then the inequality (3.2) is satisfied with the constant $s=\frac{1}{4 \delta} \exp (2 \operatorname{osc}(\hat{\Pi}))$ where

$$
\operatorname{osc}(\hat{\Pi})=\sup \hat{\Pi}-\inf \hat{\Pi}=\log \left(\frac{A^{*}}{A_{*}}\right)
$$

and this achieves the proof.

Let $\psi$ be the solution to (2.7). We prove below the following result:

Lemma 3..2. For any $\psi_{0} \in L_{M}^{2}, \psi_{0} \geq 0, \psi_{0} \not \equiv 0$ we have

$$
\left\|\psi-b \psi_{\infty}\right\|_{L^{1}(D)} \leq \frac{2}{\sqrt{A_{*}}}\left\|\psi_{0}\right\|_{L_{M}^{2}} e^{-\beta t}, \quad \forall t \geq 0
$$

where $\psi(t)=e^{-t L} \psi_{0}, b=\int_{D} \psi_{0} \mathrm{~d} q>0$ and $\beta=\delta\left(\frac{A_{*}}{A^{*}}\right)^{2}$. 
Proof. Following [12] we introduce the entropy function

$$
H(t)=\int_{D} \frac{\psi}{b} \log \left(\frac{\psi}{b \psi_{\infty}}\right) \mathrm{d} q
$$

As the above functional may not be defined for any $\psi_{0} \in L_{M}^{2}$, let us use density concept related arguments. As $\mathscr{D}(D)$ is dense in $L_{M}^{2}$ and since $\psi_{0} \geq 0$, there exists a sequence $\tilde{\psi}_{0}^{(n)} \in \mathscr{D}(D)$, $\tilde{\psi}_{0}^{(n)} \geq 0$, such that $\tilde{\psi}_{0}^{(n)} \underset{n \rightarrow+\infty}{\stackrel{L_{M}^{2}}{\longrightarrow}} \psi_{0}$. Set $\hat{\psi}_{0}^{(n)}=\tilde{\psi}_{0}^{(n)}+\frac{1}{n} M, b_{n}=\int_{D} \hat{\psi}_{0}^{(n)} \mathrm{d} q, \psi_{0}^{(n)}=\frac{\hat{\psi}_{0}^{(n)}}{b_{n}}$. It is clear that $\psi_{0}^{(n)} \underset{n \rightarrow+\infty}{\stackrel{L_{M}^{2}}{\longrightarrow}} \frac{\psi_{0}}{b}, b_{n} \underset{n \rightarrow+\infty}{\longrightarrow} b, \psi_{0}^{(n)} \in \stackrel{\circ}{P}_{1}$ and $\int_{D} \psi_{0}^{(n)} \mathrm{d} q=1$.

Let us now set $\psi^{(n)}=e^{-t L} \psi_{0}^{(n)}$, that is $\psi^{(n)}(q, t)$ is the solution to

$$
\begin{aligned}
& \frac{\partial \psi^{(n)}}{\partial t}+L \psi^{(n)}=0 \\
& \psi^{(n)}(t=0)=\psi_{0}^{(n)} .
\end{aligned}
$$

We have $\int_{D} \psi^{(n)} \mathrm{d} q=1$, for all $t \geq 0$. From the continuity in $L_{M}^{2}$ of the operator $x \mapsto e^{-t L} x$ it is clear that, for any $t>0$, we have

$$
\psi^{(n)}(t) \underset{n \rightarrow+\infty}{\stackrel{L_{M}^{2}}{\longrightarrow}} \frac{\psi(t)}{b} .
$$

Consider now the entropy functional

$$
H^{(n)}(t)=\int_{D} \psi^{(n)} \log \left(\frac{\psi^{(n)}}{\psi_{\infty}}\right) \mathrm{d} q
$$

Since conditions (B.128)-(B-129) of [12] are satisfied, we deduce from [12] that $\psi(\cdot, t) \in \stackrel{\circ}{P}_{1}$ for any $t \geq 0$. Then the hypotheses (A.126)-(A.127) of [12] are satisfied as well, and this fact one allows to obtain (see [12])

$$
\frac{d H^{(n)}}{d t}+\frac{1}{2} \int_{D}\left|\nabla\left(\frac{\psi^{(n)}}{\psi_{\infty}}\right)\right|^{2} \frac{\psi_{\infty}^{2}}{\psi^{(n)}} \mathrm{d} q=0 .
$$

With the help of Lemma $3 . .1$ we obtain

$$
4 \beta H^{(n)}(t) \leq \int_{D}\left|\nabla\left(\frac{\psi^{(n)}}{\psi_{\infty}}\right)\right|^{2} \frac{\psi_{\infty}^{2}}{\psi^{(n)}} \mathrm{d} q .
$$

It follows that

$$
\frac{d H^{(n)}}{d t}+2 \beta H^{(n)} \leq 0
$$


which implies

$$
H^{(n)}(t) \leq H^{(n)}(0) e^{-2 \beta t}, \forall t \geq 0 .
$$

As $\log z \leq z, \forall z>0$, one gets:

$$
H^{(n)}(0) \leq \int_{D} \frac{\left(\psi_{0}^{(n)}\right)^{2}}{\psi_{\infty}} \mathrm{d} q \leq \sup _{q \in D}\left(\frac{1}{A(q)}\right)\left\|\psi_{0}^{(n)}\right\|_{L_{M}^{2}}^{2}
$$

which gives, invoking (3.6), for large enough $n$

$$
H^{(n)}(t) \leq \frac{1}{A_{*}}\left\|\psi_{0}^{(n)}\right\|_{L_{M}^{2}}^{2} e^{-2 \beta t}
$$

Using Csiszar-Kullback inequality (see [12]) we get

$$
H^{(n)}(t)=\int_{D} \psi^{(n)} \log \left(\frac{\psi^{(n)}}{\psi_{\infty}}\right) \mathrm{d} q \geq \frac{1}{4}\left(\int_{D}\left|\psi^{(n)}-\psi_{\infty}\right| \mathrm{d} q\right)^{2}
$$

and with the help of (3.7) one has

$$
\int_{D}\left|\psi^{(n)}-\psi_{\infty}\right| \mathrm{d} q \leq \frac{2}{\sqrt{A_{*}}}\left\|\psi_{0}^{(n)}\right\|_{L_{M}^{2}} e^{-\beta t}, \quad \forall t \geq 0 .
$$

Letting $n \rightarrow+\infty$, using the continuous embedding $L_{M}^{2} \underset{\text { cont }}{\hookrightarrow} L^{1}(D)$ as well as the convergence property in (3.4) eventually lead to the stated result.

As already announced in Section 1., we proved in [7] that 0 is a simple eigenvalue of the operator $L$, with $\psi_{\infty}$ one of the associated eigenvectors, and that 0 is the principal eigenvalue, i.e. $\operatorname{Re}(\lambda) \geq 0$, for all eigenvalues $\lambda$ of $L$. These results are a consequence of the Krein-Rutman theorem. Actually an even stronger result can be stated:

Lemma 3..3. For any non-zero eigenvalue $\lambda$ of $L, \operatorname{Re}(\lambda)>0$.

Proof. We proceed as in [20]. Assume the contrary: say $\lambda=i \mu, \mu \in \mathbb{R}^{*}$, is an eigenvalue of $L$ with a corresponding eigenvector $\varphi^{E}=\varphi_{R e}+i \varphi_{I m}, \varphi_{R e}, \varphi_{I m} \in D(L)$. Then

$$
\begin{gathered}
L \varphi_{R e}=-\mu \varphi_{I m} \\
L \varphi_{I m}=\mu \varphi_{R e}
\end{gathered}
$$

Remark that $\varphi_{\text {Im }} \neq 0$ and $\varphi_{\text {Re }} \neq 0$.

It is clear that $\varphi(t)=e^{-t L} \varphi_{R e}$ is given by

$$
\varphi(t)=\varphi_{R e} \cos (\mu t)+\varphi_{I m} \sin (\mu t)
$$


We now prove that

$$
\varphi_{R e}, \varphi_{\text {Im }} \in X_{1}
$$

with $X_{1}$ being defined in (1.18). From equations (3.8) and (3.9) we obtain

$$
\begin{gathered}
\varphi_{R e}=B_{\alpha}\left(-\mu \varphi_{I m}+\alpha \varphi_{R e}\right) \\
\varphi_{\text {Im }}=B_{\alpha}\left(\mu \varphi_{R e}+\alpha \varphi_{\text {Im }}\right)
\end{gathered}
$$

with $\alpha>0$ large enough (see Lemma (2..2)), where $B_{\alpha}=(L+\alpha \mathrm{Id})^{-1}$. From Lemmas 3.3, 3.4 and 3.5 in [7] observe that $B_{\alpha} \in \mathscr{L}\left(L_{M}^{2}, X_{1 / 2}\right), B_{\alpha} \in \mathscr{L}\left(X_{\beta-2 / \delta}, X_{\beta}\right)$ for $1 / 2+1 /(2 \delta)<\beta<1$, and $B_{\alpha} \in \mathscr{L}\left(X_{1-1 / \delta}, X_{1}\right)$. Then (3.11) is obtained recursively.

Set now $\varphi_{0}=\varphi_{R e}+\gamma \psi_{\infty}$, with $\gamma>0$ large enough s.t. $\varphi_{0} \geq 0$ (a fact rendered possible as $\varphi_{R e} \in X_{1}$ and $\psi_{\infty} \in \stackrel{\circ}{P}_{1}$ ). Integrating equations (3.8)-(3.9) on $D$ gives

$$
\int_{D} \varphi_{I m} \mathrm{~d} q=\int_{D} \varphi_{R e} \mathrm{~d} q=0
$$

which entails

$$
\int_{D} \varphi_{0} \mathrm{~d} q=\gamma
$$

Observe now that $\varphi(t)+\gamma \psi_{\infty}=e^{-L t} \varphi_{0}$. Making use of Lemma (3..2) with $b=\gamma$ leads to $\varphi(t)+\gamma \psi_{\infty}$ converges exponentially to $\gamma \psi_{\infty}$ for $t \rightarrow+\infty$, which implies that $\varphi(t) \rightarrow 0$ for $t \rightarrow+\infty$. On the other hand, from (3.10) one gathers $\varphi(2 k \pi /|\mu|)=\varphi_{R e}$, for all $k \in \mathbb{N}$; these last facts are contradictory in nature, the proof is over.

Next, let the following space be defined as

$$
\stackrel{\circ}{L}_{M}^{2}:=\left\{\varphi \in L_{M}^{2}, \int_{D} \varphi \mathrm{d} q=0\right\}
$$

denote the closed vector subspace of $L_{M}^{2}$ functions of zero average. Then $L_{M}^{2}=\mathbb{R} \psi_{\infty} \oplus \stackrel{\circ}{2}$. In fact, for any $\varphi \in L_{M}^{2}$, the below decomposition

$$
\varphi=\frac{\int_{D} \varphi \mathrm{d} q}{\int_{D} \psi_{\infty} \mathrm{d} q} \psi_{\infty}+\left(\varphi-\frac{\int_{D} \varphi \mathrm{d} q}{\int_{D} \psi_{\infty} \mathrm{d} q} \psi_{\infty}\right)
$$

is unique.

For any $\varphi \in D(L)$ we have

$$
\int_{D}(L \varphi)(q) \mathrm{d} q=0
$$

then $L \varphi \in \stackrel{\circ}{L}_{M}^{2}$ for any $\varphi \in L_{M}^{2}$. We also have $L \psi_{\infty}=0$. Then $\mathbb{R} \psi_{\infty}$ and $\stackrel{\circ}{L}_{M}^{2}$ are invariant subspaces with respect to the operator $L$. Next, let $L_{1}$ denote the restriction of $L$ to $\mathbb{R} \psi_{\infty}, L_{2}$ the 
restriction of $L$ to $\stackrel{\circ}{L}_{M}^{2}$ with $D\left(L_{2}\right)=D(L) \cap \stackrel{\circ}{L}_{M}^{2}$. On one hand, $L_{1}$ is equal to 0 , so $\sigma\left(L_{1}\right)=\{0\}$. It is not complicated to observe that $\sigma\left(L_{2}\right)=\sigma(L)-\{0\}$. On the other, Lemma (3..3) grants the existence of $\lambda_{0}>0$ s.t. $\operatorname{Re}(\lambda) \geq \lambda_{0}$, for any $\lambda \in \sigma\left(L_{2}\right)$. Next, from Theorem 1.5.3 in [10] one sees the operator $L_{2}$ is sectorial, and there exists a constant $c \geq 0$ s.t.

$$
\begin{gathered}
\left\|e^{-t L_{2}}\right\|_{\mathscr{L}\left(\stackrel{L}{M}_{M}^{2}\right)} \leq c e^{-t \lambda_{0}}, \quad \forall t \geq 0 \\
\left\|L_{2} e^{-t L_{2}}\right\|_{\mathscr{L}\left(\stackrel{L}{L}_{M}^{2}\right)} \leq \frac{c}{t} e^{-t \lambda_{0}}, \quad \forall t>0 .
\end{gathered}
$$

We are now in a position allowing to state this paper main result:

Theorem 3..1 (Main Result). There exists a constant $c \geq 0$ such that, for any $\psi_{0} \in L_{M}^{2}$, we have the following exponential convergences:

$$
\begin{aligned}
& \left\|\psi(t)-b \psi_{\infty}\right\|_{L_{M}^{2}} \leq c e^{-t \lambda_{0}}\left\|\psi_{0}-b \psi_{\infty}\right\|_{L_{M}^{2}}, \forall t \geq 0 \\
& \left\|\psi(t)-b \psi_{\infty}\right\|_{D(L)} \leq \frac{c}{t} e^{-\lambda t}\left\|\psi_{0}-b \psi_{\infty}\right\|_{L_{M}^{2}}, \forall t>0
\end{aligned}
$$

with $b=\int_{D} \psi_{0} \mathrm{~d} q$ and $\psi$ solution to $(2.7)$.

Proof. Let us set $\zeta(t)=\psi(t)-b \psi_{\infty}$. Since $\int_{D} \psi(t) \mathrm{d} q=\int_{D} \psi_{0} \mathrm{~d} q, \forall t \geq 0$, we have $\zeta(t) \in \stackrel{\circ}{L}_{M}^{2}$, $\forall t \geq 0$. Moreover, $\zeta$ satisfies

$$
\begin{aligned}
& \frac{d \zeta}{d t}+L_{2} \zeta=0, \forall t \geq 0 \\
& \zeta(t=0)=\psi_{0}-b \psi_{\infty}
\end{aligned}
$$

Therefore, $\zeta(t)=e^{-t L_{2}}\left(\psi_{0}-b \psi_{\infty}\right)$. Use of inequalities (3.12) and (3.13) leads to the results.

Remark 3..1. Theorem (3..1) provides a stronger result than that of Lemma (3..2) because:

1. the estimates are obtained w.r.t. the stronger norm of the space $L_{M}^{2}$ or $D(L)$

2. the assumption $\psi_{0} \geq 0$ is no longer necessary (from a purely mathematical standpoint).

\section{References}

[1] J. W. Barret, C. Schwab, E. Süli. Existence of global weak solutions for some polymeric flow models. Math. Model. Meth. Appl. Sci., 15 (2005), No. 6, 939- 983.

[2] R. B. Bird, R. C. Armstrong, O. Hassager. Dynamics of Polymeric Liquids, Vol. 1: Fluid Mechanics. J. Wiley \& Sons, New York, 1987. 
[3] R. B. Bird, R. C. Armstrong, O. Hassager. Dynamics of Polymeric Liquids, Vol. 2: Kinetic Theory. J. Wiley \& Sons, New York, 1987.

[4] A. V. Busuioc, I. S. Ciuperca, D. Iftimie and L. I. Palade. The FENE dumbbell polymer model: existence and uniqueness of solutions for the momentum balance equation. Journal of Dynamics and Differential Equations, submitted, 2011.

[5] J. A. Carillo, S. Cordier, S. Mancini. A decision-making Fokker-Planck model in computational neuroscience. To appear in Journal of Mathematical Biology, 2011.

[6] L. Chupin. The FENE model for viscoelastic thin film flow. Methods Appl. Anal., 16 (2009), No. 2, 217-261.

[7] I. S. Ciuperca, L. I. Palade. The steady state configurational distribution diffusion equation of the standard FENE dumbbell polymer model: existence and uniqueness of solutions for arbitrary velocity gradients. Mathematical Models \& Methods in Applied Sciences, 19 (2009), 2039-2064.

[8] I. S. Ciupercă, L. I. Palade. On the existence and uniqueness of solutions of the configurational probability diffusion equation for the generalized rigid dumbbell polymer model. Dynamics of Partial Differential Equations, 7 (2010), 245-263.

[9] Q. Du, C. Liu, P. Yu. FENE dumbbell model and its several linear and nonlinear closure approximations. Multiscale Model. Simul., 4 (2005), No. 3, 709-731.

[10] D. Henry. Geometric Theory of semilinear parabolic equations. Lecture notes in mathematics, Vol. 840. Springer Verlag, New York, 1981.

[11] R. R. Huilgol, N. Phan-Thien. Fluid Mechanics of Viscoelasticity. Elsevier, Amsterdam, 1997.

[12] B. Jourdain, C. Le Bris, T. Lelièvre, F. Otto. Long-time asymptotics of a multiscale model for a polymeric fluid flows. Arch. Rational Mech. Anal., 181 (2006), 97-148.

[13] J. G. Kirkwood. Macromolecules, edited by P. L. Auer. Gordon and Breach, 1968.

[14] R. G. Larson. Constitutive Equations for Polymer Melts and Solutions. Butterworths, Boston, 1988.

[15] F. Lin, P. Zhang, Z. Zhang. On the global existence of smooth solution to the 2-D FENE Dumbell Model. Commun. Math. Phys., 277 (2008), 531-553.

[16] N. Masmoudi. Well-Posedness for the FENE dumbbell model of polymeric flows. Comm. Pure Appl. Math., 61 (2008), No. 12, 1685-1714.

[17] F. A. Morrison. Understanding Rheology. Oxford University Press, Oxford, 2001. 
[18] J. Nečas. Les méthodes directes en théorie des équations elliptiques. Masson, Paris, 1967.

[19] S. Cleja-Ţigoiu, V. Ţigoiu. Rheology and Thermodynamics, Part I - Rheology. Editura Universităţii din Bucureşti, Bucureşti, 1998.

[20] V. A. Volpert, A. I. Volpert. Location of spectrum and stability of solutions for monotone parabolic system. Advances in Differential Equations, 2 (1997), No. 5, 811-830.

[21] H. Zhang, P. Zhang. Local existence for the FENE-dumbbell model of polymeric fluids. Arch. Ratl. Mech. Anal., 181 (2006), 373 -400. 